\title{
Plan Piratome : Étude des procédures et moyens de décontamination
}

\author{
D. GIORDAN ${ }^{1}$
}

(Manuscrit reçu le 2 septembre 2005, accepté le 26 décembre 2005)

RÉSUMÉ Les cellules mobiles d'intervention radiologiques sont des équipes de sept sapeurs pompiers capables de prendre en charge un incident ou un accident à caractère radioactif. Un certain nombre d'unités de sapeurs pompiers ont été dotées par l'État de chaînes mobiles de décontamination. En 2004, la cellule mobile d'intervention radiologique des Yvelines a testé, trois fois, une chaîne à contamination réelle. Le technétium-99m a une période radioactive de six heures. Les victimes contaminées sont des mannequins. De nombreuses connaissances ont été acquises de ces expériences et, en corollaire, ont induites une révision des concepts et techniques opérationnels.

ABSTRACTS Piratome plan: study of the procedures of decontamination.

The firemen radiological units, created in 1980, are teams of 7 firemen able to cope with and overcome an incident or an accident of a radioactive nature. Some fire brigades have been equiped with decontamination units insuring the treatment of contaminated people. In 2004, radiological unit of Yveline's county tested the decontamination unit with real radioactive contamination three times. The technetium-99m has a half life of six hours. Contaminated victims were dummies. Much knowledge were gained from these experiments and as a result have induced a revision of operational doctrine and technics.

Key words: Piratome / CBNR Terrorism / Decontamination units / Firemen radiological rescue units

\section{Introduction}

\section{Problématique}

Un certain nombre de Services départementaux d'incendie et de secours ont été dotés par l'État d'équipements de protection des populations permettant de faire face à un accident majeur ou à un acte malveillant à caractère technologique.

Les équipements les plus marquant sont les chaînes de décontamination (Fig. 1).

Deux types de décontamination existent actuellement :

(1) la décontamination de masse que l'on pourrait qualifier de « succincte », notamment dans les armées, où l'effet recherché est de renvoyer les personnels au

\footnotetext{
1 Service départemental d'incendie et de secours des Yvelines, 78005 Versailles Cedex, France.
} 


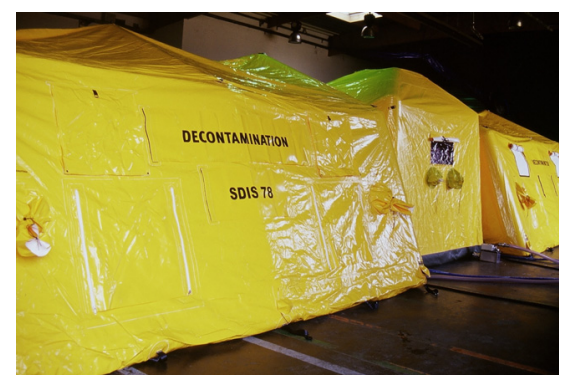

Figure 1 - Chaîne de décontamination du SDIS des Yvelines.

Decontamination unit of the Yvelines Fire Brigade.

combat le plus rapidement possible et où, du fait des tenues de protection utilisées, l'efficacité n'a pas besoin d'être optimale. L'effectif affecté aux opérations est réduit alors que le flux de personnes à décontaminer est important ;

(2) la décontamination « fine » développée dans certains services hospitaliers civils ou militaires ou de médecine du travail où une efficacité totale est recherchée. Le nombre de personnes affectées à chaque contaminé peut atteindre la dizaine et le temps de décontamination étant souvent de l'ordre de la demi heure.

Or, aujourd'hui, les services d'incendie et de secours doivent faire « vite et bien » avec un effectif optimal.

De plus, à la connaissance de l'auteur, jamais une chaîne mobile n'a été testée à contamination radioactive réelle. Il convenait, donc, de lever un certain nombre d'inconnues afin de gagner en efficacité.

Trois séries d'expériences ont été réalisées, le 28 avril, le 30 juin et le 29 septembre 2004, sur le site de l'école départementale des sapeurs pompiers des Yvelines, à Trappes et dans un vieux fort de la ceinture parisienne. Les objectifs étaient, d'abord quantitatifs :

- expériences de plusieurs modes d'organisation entre les éléments du système de prise en charge des impliqués,

- expériences de plusieurs modes d'organisation au sein des chaînes de décontamination ;

puis qualitatifs :

- étude de l'évolution de la contamination radioactive au sein des chaînes,

- étude de l'évolution de la contamination radioactive des opérateurs,

- confrontation des résultats aux modes d'organisation envisagés.

Avertissement: cet article ne se veut pas une analyse exhaustive de la problématique, mais plutôt la collection de résultats partiels, visant à enrichir la réflexion des uns et des autres, suite à un certain nombre d'expériences et d'essais. 


\section{Matériel et méthode}

\subsection{Le plan Piratome}

Le plan Piratome organise la prise en charge de personnes contaminées suite à un acte malveillant en présence de matières radioactives.

Dans un premier temps, les victimes contaminées sont regroupées au point de regroupement des victimes (PRV) où un tri s'effectue en fonction du bilan médical et de la contamination. Les personnes les plus gravement atteintes sont traitées sur place et évacuées non décontaminées vers un centre hospitalier : «l'urgence classique prime le risque radiologique $»$.

Les blessés moins gravement atteints et les simples contaminés sont décontaminés en chaîne mobile, puis,

- pour les premiers, admis dans un poste médical avancé (PMA),

- pour les seconds, pris en charge par la cellule d'urgence médico-psychologique

(Fig. 2).

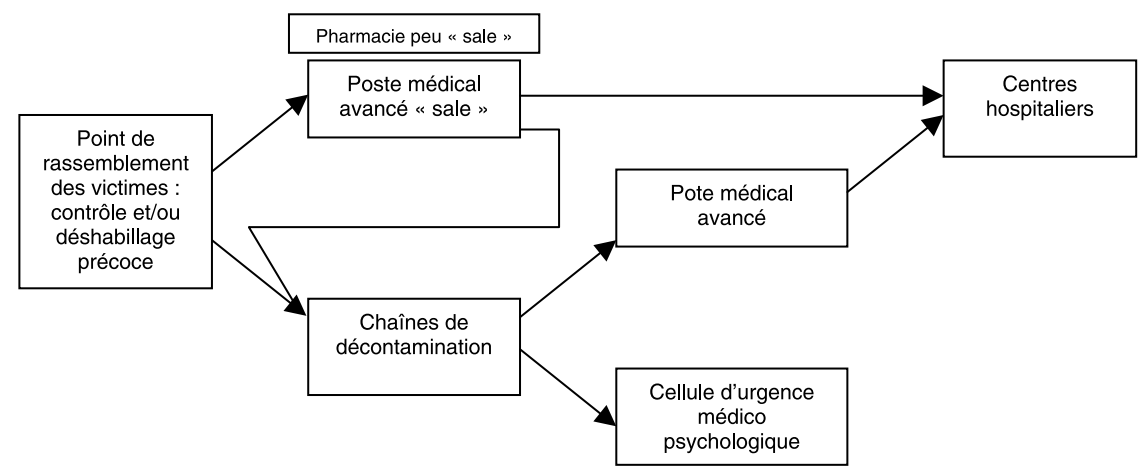

Figure 2 - Flux des victimes.

Flow of the victims.

\subsection{Schéma type d'une chaîne mobile de décontamination}

Les chaînes de décontamination mobiles s'articulent, la plupart du temps, en trois alvéoles. C'est le cas des trois chaînes testées lors des essais qualitatifs et quantitatifs : TMB Grande couronne, TMB BSPP et Utilys. Chaque alvéole a sa propre fonction (Fig. 3). 


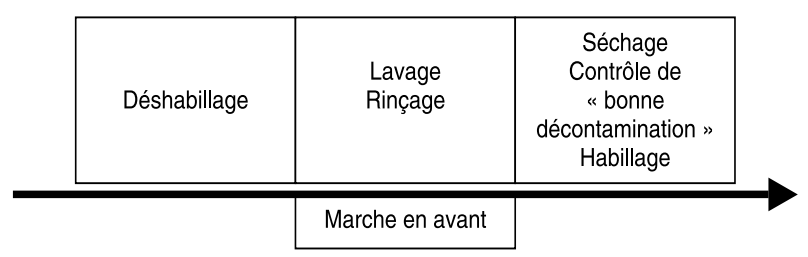

Figure 3 - Chaîne de décontamination.

Decontamination unit.

\subsection{Moyens mis en ouvre}

La manœuvre la plus importante, fin septembre, a associé deux cellules mobiles d'intervention radiologique (CMIR) 78 et BSPP (Brigade des sapeurs pompiers de Paris), une CMIR 06, 77, 91 et 95, une équipe de reconnaissance 28, 52 et 54. À ces moyens se rajoutaient une équipe d'intervention de premier échelon du CEA et trois masters gémini de l'IRSN. Des membres de la CMIC 78 (chimique) des Yvelines complétaient les effectifs. Si une seule chaîne de décontamination a été utilisée lors des deux premiers jours, c'est cinq chaînes qui le seront lors du dernier (de trois types différents : 3 de marque TMB modèle Grande couronne (77, 78 et 95), 1 TMB BSPP et 1 Utilys (54)).

C'est ainsi, une centaine de spécialistes Rad, une vingtaine de spécialistes Chim et une centaine de victimes (jeunes sapeurs pompiers et mannequins) qui y participeront.

Le radioélément utilisé était du technétium-99m. Sa courte période -6 heures - assure, naturellement, une décontamination en quelques jours de la chaîne de décontamination. L'activité utilisée a été, pour les trois expériences, de l'ordre de $200 \mathrm{MBq}$. Le technétium, livré sous forme liquide est contenu dans une fiole, ellemême renfermée dans un emballage de type A. Ces expériences se font dans le cadre de l'autorisation de détention de sources dont dispose le SDIS 78.

Le prélèvement est injecté dans une bouteille en plastique d'eau (Figs. 4 et 5) afin de permettre une dilution homogène et une bonne dissémination. Ce sont des mannequins de secourisme réformés qui jouent le rôle de victimes.

Remarque: le gros avantage, dans ce type d'expérience, d'un traceur radiologique, par rapport à un traceur chimique (comme un colorant, par exemple) est que même si le chimique n'est plus perceptible par nos sens (car trop dilué), le radiologique restera mesurable où qu'il se trouve. 


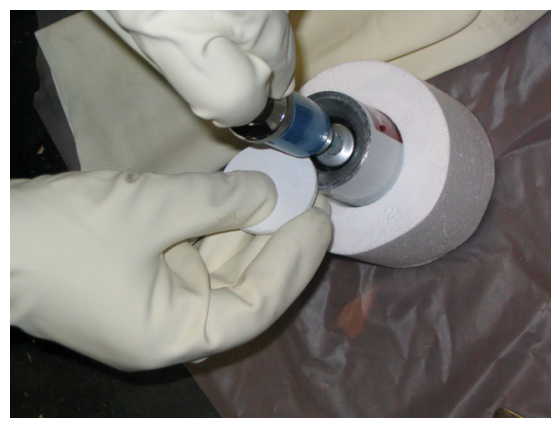

Figure 4 - Préparation de la solution de ${ }^{99 m} \mathrm{Tc}$. Preparation of the solution.

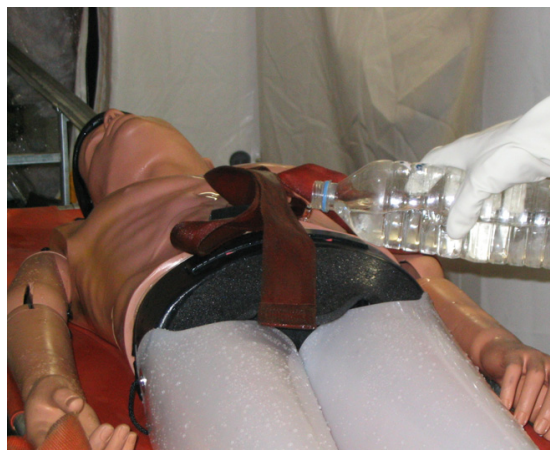

Figure 5 - Contamination d'un mannequin. Contamination of a dummy.

\subsection{Bilan qualitatif des trois jours d'expérience}

Remarque : la plupart des mesures ont été effectuées au moyen d'une sonde SX ou SX2 branchée sur un CAB ou sur un MIP (société Eurisys). En effet, lors des mesures au sol, la faible épaisseur du scintillateur NaI permet de s'affranchir en grande partie des contributions latérales qui pourraient influer sur les résultats.

\section{Résultats et discussion}

\section{1. Évolution de la contamination de la chaîne}

\subsection{1. Évolution de la contamination de l'eau utilisée}

Après passage et décontamination de mannequins dans les différentes alvéoles de la chaîne, des mesures de l'eau de décontamination sont faites (Figs. 6 et 7). Trois points de collecte ont été définis :

(1) l'eau présente sur le brancard (malgré l'inclinaison opérée sur celui-ci - qui n'est pas prévue dans la chaîne - il reste toujours une part d'humidité résiduelle),

(2) l'eau résiduelle présente sur le convoyeur (support linéaire de brancards),

(3) l'eau présente dans le bac de rétention de la douche.

Les mesures sont réalisées au moyen d'une sonde à liquide basée sur un Geiger Müller intégré dans un réceptacle en verre. Il est logique que l'eau utilisée pour la décontamination soit contaminée (Tab. I). Contaminée, elle devient contaminante. 


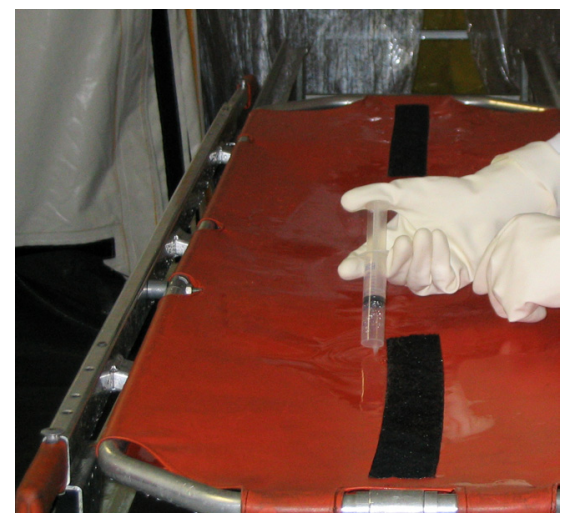

Figure 6 - Prélèvement de l'eau contaminée sur le brancard.

Take away of contamined water.

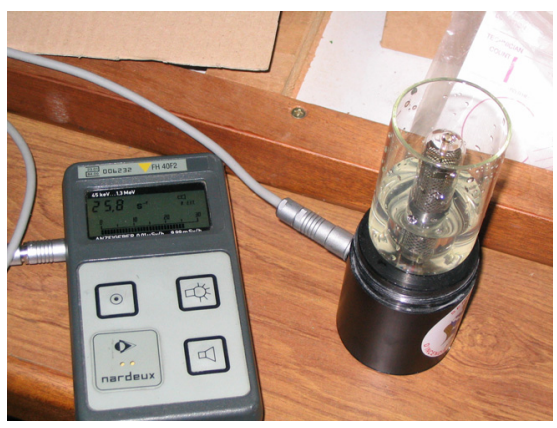

Figure 7 - Mesure de la solution prélevée. Measure of taken solution.

TABLEAU I

Résultats des mesures.

Results of measures.

\begin{tabular}{cccc}
\hline $\begin{array}{c}\text { Bruit de fond } \\
\text { (eau pure) }\end{array}$ & $\begin{array}{c}\text { Eau prélevée dans le bac } \\
\text { de la douche }\end{array}$ & $\begin{array}{c}\text { Eau prélevée sur le convoyeur } \\
\text { (rail du brancard) }\end{array}$ & $\begin{array}{c}\text { Eau prélevée sur le } \\
\text { brancard }\end{array}$ \\
\hline $0,2 \mathrm{c} / \mathrm{s}$ & $0,6 \mathrm{c} / \mathrm{s}$ & $3 \mathrm{c} / \mathrm{s}$ & $26 \mathrm{c} / \mathrm{s}$ \\
\hline
\end{tabular}

Le brancard et le convoyeur représentent un vecteur de dissémination de contamination.

1. La zone propre située dans le prolongement de la douche ne le restera pas longtemps sauf à trouver une solution pour éviter le ruissellement depuis le brancard et le long du convoyeur.

2. De plus, la comparaison entre le fonctionnement des chaînes Utilys et TMB a permis de mettre en exergue l'importance, pour la prévention de la dissémination de la contamination, de la surélévation du caillebotis présent sur les douches.

Ainsi, à mesure du traitement des victimes, il faut s'attendre à une contamination croissante de la chaîne entraînant une augmentation du taux de comptage observé. 


\subsection{2. Évolution de la contamination de la troisième alvéole}

Pourtant, la troisième alvéole est censée rester la plus propre possible. Or, les comptages démontrent qu'elle augmente avec le nombre de victimes traitées et qu'à terme, elle risque d'être trop souillée.

Ce type de comptage interdit tout retour en arrière de personnes mal décontaminées. Le respect de la marche «en avant» impose de mettre en place une chaîne en aval des chaînes chargées du traitement initial afin de recueillir les personnes encore contaminées : c'est le concept de la « $3^{\mathrm{e}}$ chaîne ». Deux chaînes primaires pour une chaîne secondaire en soutien des deux précédentes.

\subsection{3. Évolution du taux de comptage dans la $3^{e}$ alvéole}

Le contrôle de «bonne décontamination » en sortie de chaîne (Fig. 8) doit se faire dans un environnement exempt de toute radioactivité autre que naturelle.

Or, on observe une augmentation du bruit de fond ambiant, liée aux différents éléments contaminés :

- contamination de la douche,

- contamination des canalisations et pompes,

- contamination des citernes souples,

- présence des vêtements contaminés,

- contamination des sauveteurs,

- contamination des victimes se présentant en chaîne.

Il ressort de ces différents points que le contrôle de «bonne décontamination » prévu dans la troisième partie de la chaîne, depuis une cinquantaine d'année, est illusoire avec ce type d'équipement.

Plusieurs conclusions peuvent en être tirées de ces points :

(1) les citernes souples doivent pouvoir être placées à une quarantaine de mètres des chaînes de décontamination. Elles doivent être installées, le collecteur orienté à l'opposé des chaînes car c'est la partie qui présente le taux de comptage le plus fort ;

(2) pour faciliter un bon contrôle des personnes et pour diminuer l'exposition des sauveteurs, il faut prévoir un ou deux sauveteurs chargés de gérer et d'évacuer les vêtements en temps réel ;

(3) la solution la plus simple consiste à délocaliser le contrôle. Plusieurs solutions sont envisageables :

- éloigner de quelques mètres le point de mesures (par exemple en accolant une autre tente en sortie de chaîne), 


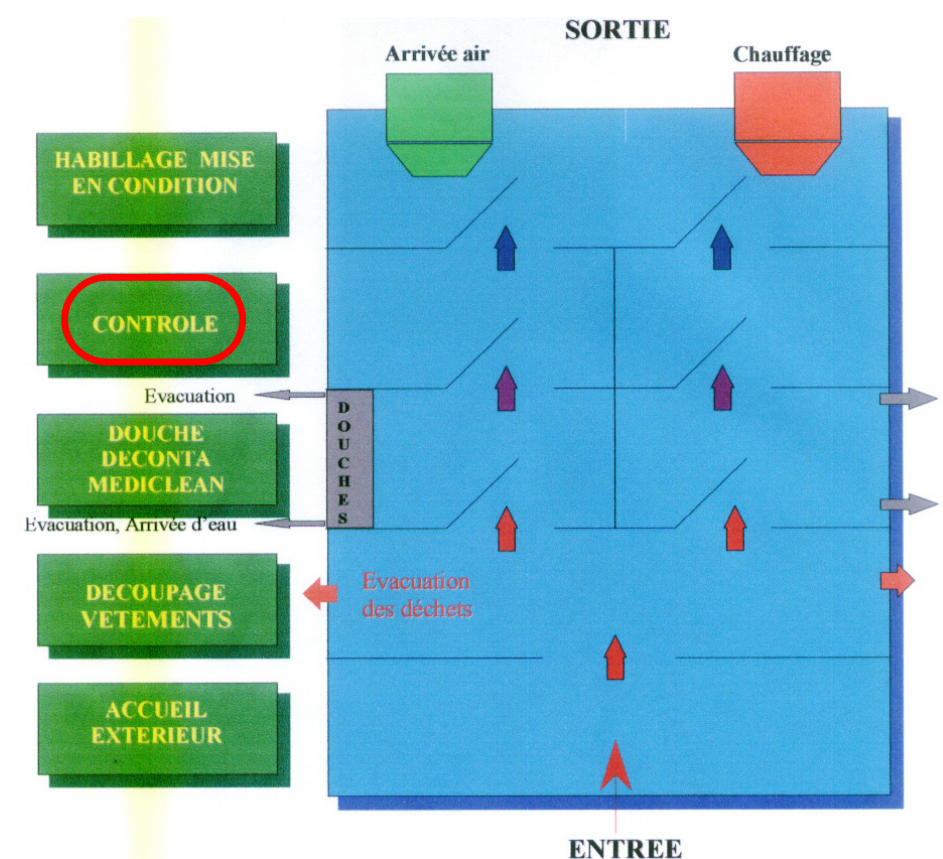

Figure 8 - Organisation envisagée, depuis 50 ans, de la chaîne de décontamination. Envisaged organization, since 50 years, of the decontamination unit.

- adosser la chaîne à un bâtiment pouvant accueillir, à distance et derrière un mur, le contrôle,

- rhabiller les personnes en sortie de chaîne, sans les contrôler et effectuer ce dernier dans un nouveau PRV situé en entrée de la $3^{\mathrm{e}}$ chaîne pouvant accueillir les personnes mal décontaminées;

(4) selon la nature du radioélément, un suivi régulier du débit de dose semble une nécessité. Le débit de dose ne devrait jamais être trop important ; par contre, le temps passé en chaîne, lui, risque de l'être.

Remarque : un acheteur averti de chaine de décontamination devrait demander que soit intercalé un long tunnel (où le séchage peut avoir lieu) entre la sortie de la douche et le lieu de contrôle de la contamination et de rhabillage (la personne le remontant nue). 


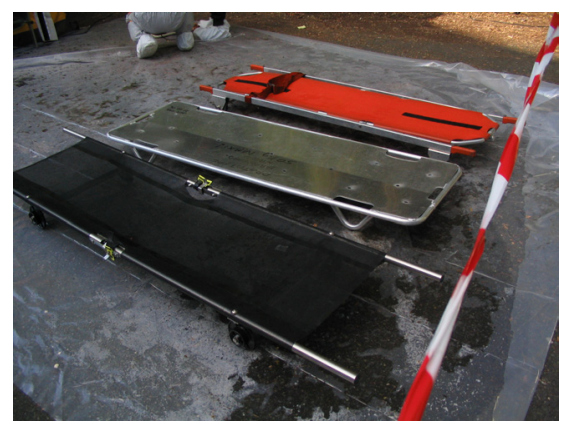

Figure 9 - Les 3 brancards testés. The 3 tested stretchers.

\subsection{4. Évolution de la contamination des brancards}

La contamination résiduelle des brancards est un point intéressant à étudier. En effet, il faut savoir, après rinçage de la victime, si ceux-ci restent contaminés.

Il a été vu, plus haut, que l'eau stagnant sur le brancard reste contaminée, malgré le rinçage à grande eau de la victime et surtout l'élimination du maximum d'eau résiduelle. Dans ce cadre, l'utilisation de brancard ajouré peut se révéler une solution qui, toutefois, risque de se révéler insuffisante tant la contamination arrive, aisément, à se nicher partout.

Trois types de brancards sont utilisés (Fig. 9) : un brancard de VSAV normal, un brancard expérimental développé par le SDIS 77 en inox avec de gros trous, un brancard commercialisé avec la chaîne utilys réalisé avec un treillis à petites mailles.

Ils sont successivement testés dans des conditions expérimentales reproductibles :

- contamination du mannequin,

- mesures sur le mannequin contaminé,

- lavage et rinçage du mannequin (6 min),

- mesures sur et sous le brancard après décontamination du mannequin.

La contamination du brancard semble être directement fonction de la quantité d'eau restant sur celui-ci :

- la toile du brancard classique des VSAV, malgré élimination de l'eau résiduelle par inclinaison forte - vers les pieds de celui-ci, retient une part d'humidité contaminée. Le taux de comptage est fort,

- de fines gouttelettes d'eau sont retenues dans les mailles du brancard de la société Utilys. Le taux de comptage, bien que bas, est supérieur au bruit de fond, 
- la forme incurvée du brancard expérimental développé par le SDIS de Seine et Marne, sa matière drainante et ses trous permettent d'éviter de retenir de l'humidité. Le taux de comptage est conforme au bruit de fond.

La conclusion principale est qu'en tout état de cause, que ce soit

- pour permettre à un brancard adapté de rester en douche

ou

- pour limiter le transfert de l'humidité contaminée,

un changement de brancard en sortie de douche paraît nécessaire à un contrôle efficace de la bonne décontamination.

\subsection{5. Évolution de la contamination des sauveteurs}

Le sauveteur le plus exposé au risque de contamination est le déshabilleur qui est chargé de basculer la victime invalide. La mesure a été faite au niveau des mains et du torse hors valeur ambiante de la tente de déshabillage (Tab. II).

TABLEAU II

Résultats des mesures sur le sauveteur.

Results of measure on the rescuer.

\begin{tabular}{cc}
\hline & Taux de comptage (c/s) \\
\hline Bruit de fond & 7 \\
$1^{\text {re }}$ victime & 15 \\
$2^{\mathrm{e}}$ victime & 60 \\
$3^{\mathrm{e}}$ victime & 125 \\
$4^{\mathrm{e}}$ victime & 160 \\
\hline
\end{tabular}

(1) Dans le cadre d'une intervention réelle, les sauveteurs seront fortement contaminés (ils seront donc irradiés et contaminant). L'organisation doit prévoir une prise en charge pour eux, leur permettant de se contrôler, de se déshabiller et de se décontaminer facilement.

(2) Le port de la TLD en chaîne de décontamination doit permettre aux membres des services de secours de décontaminer leurs propres vêtements en se rinçant sous la douche. N'oublions pas que les vêtements contaminés irradient en permanence.

(3) La notion de sauveteur «non contaminé » pour la manutention des victimes trouve, ici, toute sa pertinence

(4) La logistique «tenues » sera une fonction essentielle de la protection des intervenants. 


\subsection{Remarques relatives à d'autres entités de la zone contaminée}

Les médecins, infirmiers, pharmaciens et vétérinaires sapeurs pompiers constituent le Service de santé et de secours médical (SSSM) des SDIS. Leur implication dans les secours à caractère technologiques est forte. Ainsi, ils trouvent toute leur place dans le cadre des actions de décontamination et de secours aux contaminés. On les retrouvera dans les deux entités suivantes : la notion de PMA « sale » et la notion de pharmacie « sale ».

\subsubsection{La notion de PMA « sale »}

Lors des interventions en présence de sources de rayonnements ionisants, le « risque classique » prime toujours sur le « risque radiologique ». C'est pourquoi, il est probable, qu'en cas d'événement radiologique, il faudra mettre en place un poste médical dans la zone contrôlée (en plus de celui prévu en aval des chaînes de décontamination). Il sera contaminé par les allers et les retours. À ce titre, il était intéressant d'étudier l'évolution de la contamination et de voir quelle organisation pourrait permettre de limiter sa dissémination.

Ainsi, le transfert de contamination aux membres du SSSM et secouristes s'est révélé relativement limité - mais, il s'agissait d'un liquide contaminant et non de poussières ! La complémentarité entre équipes médicales et spécialistes en risques radiologiques s'est révélée très efficace.

L’organisation du PMA sur la base de deux zones séparées par une délimitation au sol :

- $\quad$ sale : 2 à 4 brancards

- propre : 2 à 4 brancards

semble pertinente. Afin d'obtenir une bonne cinétique de traitement des victimes, il faut affecter 4 personnes par brancard contaminé propre ou sale.

Les actions médicales et l'emballage en vue d'une évacuation directe des urgences absolues sont suivies pas à pas par les radioprotectionnistes.

\subsubsection{La notion de pharmacie «peu sale »}

Un problème récurent est celui de la préparation des médicaments. En effet, il n'est pas envisageable de laisser des membres du Service de santé et de secours médical travailler, dans le PMA «sale », sans tenue. Or, il n'est pas possible de préparer des perfusions, des plateaux d'intubation ou des injections en portant des gants. Les préparations doivent tenir compte des pathologies, mais également du poids et de l'âge de la victime. 
Face à ce problème, deux options sont donc possibles :

(1) assurer les préparations au niveau du sas «intervenants » et faire acheminer celles-ci au PMA sale. Testée, cette solution s'avère trop lourde en allers et retours pour pouvoir être réellement efficace ;

(2) mettre en place une pharmacie «peu sale» à proximité du PMA sale où les membres du SSSM pourront préparer l'ensemble du matériel nécessaire avec une tenue de protection allégée - après l'avoir rejointe en tenue de protection complète.

Des pharmaciens, infirmiers et secouristes devront intégrer cette pharmacie.

\section{Bilan quantitatif des trois jours d'expérience}

\subsection{Prise en charge des victimes - analyse des flux en chaîne}

Au-delà de la technique d'activation des chaînes, a été étudiée également, le 28 avril et le 29 septembre, l'organisation globale de la décontamination. Un centre de tri (activé au niveau du PRV) et une chaîne ont été installés afin d'accueillir des figurants valides et invalides. Il fallait étudier l'organisation la plus efficace, particulièrement en matière de débits des chaînes

Trois modes d'organisation (Tab. III) ont été testés et le temps mesuré :

(1 et 2) l'organisation telle qu'elle est prévue par la littérature avec, en chaîne, $1 /$ une douche (modèle TMB),

2/ deux douches (modèle Utilys) ;

(3) une organisation privilégiant un déshabillage précoce au niveau du PRV et libérant, ainsi, du temps d'utilisation de chaîne (modèle TMB).

Afin de rendre les différentes expériences comparables des règles strictes ont été fixées :

- temps de comptage en centre de tri fixé à $2 \mathrm{~min}$,

- temps de « mouillage + lavage »: $3 \mathrm{~min}+$ temps de « rinçage »: $3 \mathrm{~min}$.

Pour les chaînes à une seule douche, cela représente 6 min dans cette dernière (goulot d'étranglement). Il s'agit, en fait, de ce qui nous appellerons le « temps incontournable ».

L'échantillon est fixé, par chaîne, à 15 à 20 valides et 4 à 6 invalides. Les temps nécessaires sont mesurés (Tab. IV).

(1) Le déshabillage précoce donne de bons résultats, essentiellement pour les victimes invalides.

(2) la seconde organisation (avec une division du travail - deux douches - dérivée du temps « incontournable » le plus long) permet un gain important sur le débit des chaînes, particulièrement pour les personnes valides. 


\section{TABLEAU III}

Organisations quantitatives testées.

Quantitative tested organizations.

\begin{tabular}{llll}
\hline Lieu & \multicolumn{1}{c}{ Actions menées (TMB) } & \multicolumn{1}{c}{ Actions menées (Utilys) } & \multicolumn{1}{c}{ Actions menées (TMB) } \\
\hline & \multicolumn{1}{c}{ Expérience 1 } & \multicolumn{1}{c}{ Expérience 2 } & \multicolumn{1}{c}{ Expérience 3 } \\
& (TMB déshabillage en chaîne) & (Utilys déshabillage en chaîne) & (TMB déshabillage précoce) \\
\hline PRV & Contrôle & Contrôle & $\begin{array}{l}\text { Contrôle } \\
\text { Déshabillage }\end{array}$ \\
& & & Rhabillage en vêtements jetables \\
Chaîne & Déshabillage & Déshabillage & Déshabillage \\
& Lavage - rinçage (1 douche) & Lavage dans douche 1 & Lavage - rinçage (1 douche) \\
& & Rinçage dans douche 2 & \\
& Séchage & Séchage & Séchage \\
& Contrôle & Contrôle & Contrôle \\
& Rhabillage & Rhabillage & Rhabillage \\
\hline
\end{tabular}

TABLEAU IV

Résultats des trois organisation testées.

Results of the three tested organizations.

\begin{tabular}{|c|c|c|c|c|c|c|}
\hline & \multicolumn{2}{|c|}{$\begin{array}{c}\text { Expérience 1 } \\
\text { (TMB déshabillage } \\
\text { en chaîne) }\end{array}$} & \multicolumn{2}{|c|}{$\begin{array}{c}\text { Expérience 2 } \\
\text { (Utilys déshabillage } \\
\text { en chaîne) }\end{array}$} & \multicolumn{2}{|c|}{$\begin{array}{c}\text { Expérience } 3 \\
\text { (TMB déshabillage } \\
\text { précoce) }\end{array}$} \\
\hline & $\begin{array}{l}\text { Victime } \\
\text { valide }\end{array}$ & $\begin{array}{l}\text { Victime } \\
\text { invalide }\end{array}$ & $\begin{array}{l}\text { Victime } \\
\text { valide }\end{array}$ & $\begin{array}{l}\text { Victime } \\
\text { invalide }\end{array}$ & $\begin{array}{l}\text { Victime } \\
\text { valide }\end{array}$ & $\begin{array}{l}\text { Victime } \\
\text { invalide }\end{array}$ \\
\hline Transfert du brancard & & $1 \mathrm{~min}$ & & $1 \mathrm{~min}$ & & $1 \mathrm{~min}$ \\
\hline Déshabillage & $3 \min 30$ & $5 \min 30$ & $3 \min 30$ & $5 \min 30$ & $\begin{array}{l}1 \min 30 \\
\text { (pyjama) }\end{array}$ & $\begin{array}{l}0 \text { min } \\
\text { (déjà déshabillé) }\end{array}$ \\
\hline $\begin{array}{l}\text { Aspersion, savonnage } \\
\text { et lavage } \\
\text { (fixé par convention) }\end{array}$ & $3 \mathrm{~min}$ & $3 \mathrm{~min}$ & $3 \mathrm{~min}$ & $3 \mathrm{~min}$ & $3 \mathrm{~min}$ & $3 \mathrm{~min}$ \\
\hline $\begin{array}{l}\text { Rinçage } \\
\text { (fixé par convention) }\end{array}$ & $3 \mathrm{~min}$ & $3 \mathrm{~min}$ & $3 \mathrm{~min}$ & $3 \mathrm{~min}$ & $3 \mathrm{~min}$ & $3 \mathrm{~min}$ \\
\hline Transfert brancard & & $1 \mathrm{~min}$ & & $1 \mathrm{~min}$ & & $1 \mathrm{~min}$ \\
\hline Essuyage & $1 \min 30$ & $2 \min 30$ & $1 \min 30$ & $2 \min 30$ & $1 \min 30$ & $2 \min 30$ \\
\hline Contrôle & $2 \min$ & $2 \min$ & $2 \min$ & $2 \min$ & $2 \min$ & $2 \min$ \\
\hline Habillage - emballage & $1 \min 30$ & $1 \mathrm{~min}$ & $1 \min 30$ & $1 \mathrm{~min}$ & $1 \min 30$ & $1 \mathrm{~min}$ \\
\hline Pose couverture & & $0 \min 30$ & & $0 \min 30$ & & $0 \min 30$ \\
\hline $\begin{array}{l}\text { Imprévus + transferts } \\
\text { divers }\end{array}$ & $2 \min 30$ & $2 \min 30$ & $2 \min 30$ & $2 \min 30$ & $2 \min 30$ & $2 \min 30$ \\
\hline $\begin{array}{l}\text { Temps moyen } \\
\text { observé }\end{array}$ & $17 \mathrm{~min}$ & $22 \mathrm{~min}$ & $17 \mathrm{~min}$ & $22 \mathrm{~min}$ & $15 \mathrm{~min}$ & $16 \min 30$ \\
\hline $\begin{array}{l}\text { Temps minimum } \\
\text { observé }\end{array}$ & $15 \min 30$ & $16 \min 30$ & $16 \min 30$ & $16 \min 30$ & $14 \mathrm{~min} 30$ & $16 \mathrm{~min}$ \\
\hline $\begin{array}{l}\text { Temps maximum } \\
\text { observé }\end{array}$ & $23 \mathrm{~min}$ & $35 \mathrm{~min}$ & $17 \mathrm{~min}$ & $33 \mathrm{~min}$ & $17 \mathrm{~min}$ & $18 \mathrm{~min}$ \\
\hline
\end{tabular}


(3) Éléments complémentaires :

- déshabillage : s'il n'est effectué que par un seul découpeur, le temps dévolu au déshabillage est plus que doublé. La meilleure des solutions consiste à placer, face à face, les deux sauveteurs, de part et d'autre du contaminé,

- déshabillage : la localisation exacte de la contamination permet de gagner du temps lors du déshabillage (d'où l'importance d'une fiche de suivi),

- pour tout travail en chaîne : le temps est directement fonction de la masse et de la taille de la victime.

(4) La décontamination partielle n'est pas envisagée, il faudra étudier si elle est de nature à faire gagner du temps dans le traitement global du flux et si elle ne pose pas trop de problèmes dans le cadre d'une opération de décontamination de masse.

Remarque : en outre, un déshabillage précoce, au PRV, permettra :

(1) de soustraire rapidement les contaminés à une partie du toxique,

(2) de donner l'impression aux victimes qu'elles sont prises en compte plus rapidement par les sauveteurs,

(3) de répondre, au moins partiellement, à l'objectif fixé par les pouvoirs publics «Il est nécessaire de décontaminer les victimes dans les meilleurs délais ».

\subsection{Organisation des secours}

\subsubsection{Notion d'«équipage "}

L'effectif nécessaire à la mise en œuvre de chaînes de décontamination doit permettre de respecter la «marche en avant» vers la «non-contamination» (Tab. V). Ainsi, par exemple, un sauveteur dont les gants sont contaminés ne devra plus toucher à une victime décontaminée.

Il faudra des relèves régulières. Une relève toutes les deux heures, si la température de la chaîne ne dépasse pas les $20{ }^{\circ} \mathrm{C}$ semble un minimum. Si la chaleur est plus forte, des relèves plus nombreuses semblent nécessaires.

(1) L'ensemble de ces éléments milite donc pour deux ou trois équipes de 20 personnes, par chaîne, travaillant en alternance (à la manière des équipages de sous-marins).

(2) Le chef de chaîne doit être l'interface privilégiée entre tous, tout en restant en liaison avec le chef de plusieurs chaînes. Son positionnement est donc un élément capital, du fait du bruit et du cloisonnement de chaque ligne. 


\section{TABLEAU V}

«Équipage » des chaînes de décontamination. "Crew" of the decontamination unit.

\begin{tabular}{|c|c|c|}
\hline & Fonction & Statut \\
\hline \multicolumn{3}{|l|}{ Ligne invalide * } \\
\hline Déshabillage invalide & $\begin{array}{l}5: \quad 1 \text { chef «propre » } \\
2 \text { déshabilleurs « sales » } \\
2 \text { ripeurs «propres » }\end{array}$ & $\begin{array}{l}\text { RCh } 2 \text { ou Rad } 2 \\
\text { Sans qualification particulière } \\
\text { Sans qualification particulière }\end{array}$ \\
\hline Douchage invalide & $\begin{array}{l}3: \quad 1 \text { laveur « sale » } \\
1 \text { laveur « sale » } \\
1 \text { rinceur « propre » }\end{array}$ & $\begin{array}{l}\text { RCh } 2 \text { ou Rad } 2 \\
\text { Sans qualification particulière } \\
\text { Sans qualification particulière }\end{array}$ \\
\hline Séchage invalide & $\begin{array}{l}3: \quad 1 \text { sécheur ripeur «propres » } \\
2 \text { contrôleurs ripeurs « propres » }\end{array}$ & $\begin{array}{l}\text { Sans qualification particulière } \\
\operatorname{Rad} 2\end{array}$ \\
\hline \multicolumn{3}{|l|}{ Ligne valide } \\
\hline Déshabillage valide & $\begin{array}{l}2: \quad 1 \text { déshabilleur «sale » } \\
1 \text { déshabilleur « sale » }\end{array}$ & $\begin{array}{l}\text { RCh } 2 \text { ou Rad } 2 \\
\text { Sans qualification particulière }\end{array}$ \\
\hline Douchage valide & 0 & \\
\hline Séchage valide & $\begin{array}{l}3: \quad 1 \text { sécheur «propre » } \\
2 \text { contrôleurs «propres » }\end{array}$ & $\begin{array}{l}\text { Sans qualification particulière } \\
\operatorname{Rad} 2\end{array}$ \\
\hline \multicolumn{3}{|l|}{ Autres } \\
\hline Commandement et soutien & $\begin{array}{l}4: \quad 1 \text { chef de chaîne } \\
1 \text { secrétaire } \\
1 \text { logisticien } \\
1 \text { gestionnaire des déchets }\end{array}$ & $\begin{array}{l}\text { RCh } 3 \text { ou Rad } 3 \\
\text { Sans qualification particulière } \\
\text { Conducteur EP, chauffagiste ou } \\
\text { électricien, RCh } 2 \text { ou Rad } 2 \\
\text { Sans qualification particulière }\end{array}$ \\
\hline
\end{tabular}

\subsubsection{Suivi dosimétrique, injection et sortie de la zone sale}

Rappelons, qu'au terme du code de la santé publique, la responsabilité en matière de justification, d'optimisation et de suivi de la dosimétrie pèse sur le Directeur des opérations de secours.

Les trois manœuvres ont démontré que l'injection de personnel habillé et portant son dosimètre est une action longue. En effet, l'enregistrement préalable est une nécessité, au moins à partir du moment où l'organisation est mise en place. De plus, le temps de sortie est très long: essentiellement la vérification de la contamination, mais également déshabillage, décontamination éventuelle et contrôle de la dosimétrie.

(1) Il faudra :

- assurer l'enregistrement du temps d'exposition, des doses, etc. de chaque intervenant,

- dimensionner suffisamment les effectifs de l'équipe chargée d'assurer le suivi dosimétrique de plusieurs centaines d'intervenants, 
- positionner cette équipe à un endroit calme et surtout pas à l'intérieur d'une CMIR,

- en cas de succession de plusieurs équipes dans la même chaîne, il faudra que les relèves et les sorties soient organisées par un coordinateur.

(2) L'injection en une fois d'une relève est de nature à ralentir l'entrée en zone les plus rapides attendant les plus lents. Il faudra tester injection et remplacement au fur et à mesure de la disponibilité des équipiers de relève.

\section{Conclusion}

Le point le plus important est de mentionner que ces expériences ne sont qu'une étape. Il reste encore un certain nombre de questions en suspend. Toutefois, les éléments déjà collectés devraient permettre d'améliorer la capacité opérationnelle des secours.

La fixation d'un objectif de décontamination à atteindre est une nécessité, dès le début de l'intervention. Elle conditionne toute l'organisation : qu'est-ce qui est tolérable, qu'est-ce qui ne l'est pas ? Ce niveau est le point de rencontre entre les nécessités sanitaires et médicales et les possibilités techniques.

En effet, il n'y a pas de réponse unique à l'événement, il n'y a pas de méthode universelle : de nombreux paramètres impactent le choix de la doctrine :

- nature chimique du polluant,

- forme physique du polluant,

- conditions météo,

- nombres de personnes impliquées,

- nombres de personnes blessées,

- topographie,

- ressources de la zone d'intervention,

- capacité de montée en puissance,

- etc.

Les expériences et réflexions à mener dans les prochains mois porteront sur les améliorations en matière d'équipement, notamment :

- l'utilisation de «brancards cuillères» ne fixant pas la contamination et facilitant la manutention des victimes,

- le développement d'un portique portable de contrôle permettant d'améliorer la fluidité du contrôle et déclenchant avant l'habituelle énergie de $60 \mathrm{keV}$ des scintillateurs plastiques,

- les conséquences de la contamination des tenues de protection à port permanent. 
D'autres voies devront être également explorées telles que :

- l'influence de la décontamination partielle sur les flux,

- l'influence de l'allègement de la protection en fonction du polluant.

\begin{abstract}
Abréviations et termes professionnels :
BSPP : brigade des sapeurs pompiers de Paris,

CEA : Commissariat à l'énergie atomique,

CMIC : cellule mobile d'intervention chimique,

CMIR : cellule mobile d'intervention radiologique,

IRSN : Institut de radioprotection et de sûreté nucléaire,

PMA : poste médical avancé,

PRV : point de regroupement des victimes,

SDIS : service départemental d'incendie et de secours,

SSSM : service de santé et de secours médical,

TLD : tenue légère de décontamination,
\end{abstract}

VSAV : véhicule de secours aux asphyxiés et aux victimes (ambulance des sapeurs pompiers),

Conducteur EP : conducteur engin pompe (conducteur d'engin d'incendie),

$\operatorname{Rad} 1,2,3$ ou 4 : sapeur pompier titulaire des unités de valeurs spécialisées Rad 1, 2,3 ou 4 ,

Rch 1, 2, 3 ou 4 : sapeur pompier titulaire des unités de valeurs spécialisées RCh $1,2,3$ ou 4 . 